Ulrich Deinet

\title{
Kinder und Jugendliche aus Sicht der Einrichtungen der Offenen Kinder- und Jugendarbeit: Erste Ergebnisse des Projekts „Neustart der OKJA in NRW“
}

Als Kindheits- und Jugendforscher an der Hochschule Düsseldorf im Fachbereich Sozial- und Kulturwissenschaften befasse ich mich seit Jahren mit den (veränderten) Lebenswelten von Kindern und Jugendlichen im öffentlichen Raum aber auch in Institutionen wie der Schule oder Einrichtungen der Kinder- und Jugendarbeit oder auch in Shopping Malls. Die COVID-19-Pandemie hat das Leben der Kinder und Jugendlichen nachhaltig verändert und auch für pädagogische Institutionen zu ungeahnten Herausforderungen geführt, die ich besonders im Bereich der Offenen Kinder- und Jugendarbeit aktuell untersuche.

Im Folgenden werden Zwischenergebnisse aus dem Forschungsprojekt „Neustart der Offenen Kinder- und Jugendarbeit in NRW in der Corona-Zeit“ präsentiert, das im Auftrag des Jugendministeriums Nordrhein-Westfalen in Kooperation mit den beiden Landesjugendämtern des Landes durchgeführt wird, Laufzeit von Juni 2020 bis Ende 2021. Unsere Einschätzungen beruhen auf fünfzig qualitativen Interviews ${ }^{1}$ mit vierundzwanzig Einrichtungen der Offenen Kinder- und Jugendarbeit (OKJA) in NRW und der Text verwendet Elemente unseres Zwischenberichts vom Frühjahr 2021 (Deinet/Sturzenhecker 2021).

\section{Situation der Kinder und Jugendlichen}

Das Bild, das die Fachkräfte von der Situation der Kinder und Jugendlichen beschrieben, war insgesamt recht differenziert, was u. a. an der vielfältigen Auswahl an teilnehmenden Einrichtungen lag, die sich in den örtlichen Bedingungen, den Zielgruppen und Schwerpunkten unterscheiden.

1 Auf der Basis dieser Interviews wird ein Fragebogen entwickelt, mit dem in einer quantitativen Online-Vollbefragung im Frühjahr 2021 etwa 2.400 Einrichtungen der OKJA in NRW untersucht werden.

Ә Open Access. (C) 2022 Ulrich Deinet (c))BY-NC-ND Dieses Werk ist lizenziert unter der Creative Commons Attribution-NonCommercial-NoDerivatives 4.0 Lizenz.

https://doi.org/10.1515/9783110760361-005 
In Bezug auf das Zuhause und die familiäre Situation wurden „sehr unterschiedliche“ Situationen geschildert, je nach den vorhandenen Ressourcen und Kontexten. Abhängig von der Wohnsituation, z. B. einen Ort mit Privatsphäre zu haben, technischer Ausstattung mit Internet und Endgeräten, zeitlichen Ressourcen der Eltern, Unterstützungsmöglichkeiten durch die Eltern bei schulischen Aufgaben, finanzieller Sicherheit, Kontakten zu weiteren Bezugspersonen wie u. a. Lehrerinnen und Lehrern oder Freundinnen und Freunden, wurden die von den Kindern und Jugendlichen beschriebenen Situationen teilweise als „ok“, teilweise auch als „stressig“, „eingeengt und kontrolliert“ wiedergegeben. Hier ist also kein eindeutiges Bild der Sicht der Fachkräfte über die Lage ihrer Zielgruppen zu bestimmen.

Die schulische Situation der Kinder und Jugendlichen wurde durch die Fachkräfte als abhängig vom Engagement von Seiten der Schulen und einzelnen Lehrkräften beschrieben. Mit Blick auf die zu bewältigenden Aufgaben und die Schwierigkeit, sich Sachverhalte selbst aneignen zu müssen, gaben die meisten Befragten an, dass diese eine große Last für die Kinder und Jugendlichen darstellten. Deshalb suchten die Kinder Hilfe bei Eltern oder, falls nicht möglich, den Fachkräften. Teilweise wurden zudem Sorgen und großer Druck bezüglich Schulleistungen, Versetzung oder der Suche nach Ausbildungs- und Praktikumsplätzen beschrieben. Neben positiven Ausnahmen hat sich gesamt gesehen die schulische Situation verschlechtert und es wird davon ausgegangen, dass bestimmte Gruppen von Kindern und Jugendlichen (weiter) abgehängt werden. Verschlechtert habe sich aus Sicht der Fachkräfte beispielsweise die Situation der Kinder und Jugendlichen mit (Flucht)Migrationshintergründen und in finanziellen Notlagen. Beiden fehlten technische und soziale Ressourcen. Die schulische Situation nach dem ersten Lockdown wird eher als „chaotisch“, auch als negativ und belastend dargestellt und führte durch Intransparenz der Corona-Regelungen sowie der schulischen Anforderungen und Leistungsmöglichkeiten bei vielen Schülerinnen und Schülern zu Unsicherheiten.

Es wird beschrieben, dass durch den Wegbruch von Schulzeiten und Freizeitgestaltung den Kindern und Jugendlichen ein regelmäßiger, fester Tagesablauf bzw. Tagesrhythmus (teilweise sehr deutlich) zu fehlen schien. Freizeitaktivitäten beschränkten sich hauptsächlich auf private Treffen zuhause, die Nutzung von Social Media und digitalen Spielen. Bei weiterer Öffnung des öffentlichen Raumes kam es zur Wiederaufnahme von Aktivitäten, die draußen möglich sind, wie Fußballspielen. Von Fachkräften aus dem ländlichen Raum wurde beobachtet, dass insbesondere Jugendliche auf wenig frequentierte bzw. „kontrollierte“ Orte außerhalb von Wohnungen und Einrichtungen, auch in der Natur auswichen.

Insgesamt beschrieben die Fachkräfte einen hohen Gesprächsbedarf der Kinder und Jugendlichen während des Lockdowns und bei der Wiedereröffnung der 
Einrichtungen im Sommer, z. B. zu schulischen Themen (siehe oben). Konkrete Beratungsanfragen seien hingegen weniger geworden.

Die Gefühlslagen der Kinder und Jugendlichen wurden eher negativ beschrieben. In den Interviews ist u. a. die Rede von Ärger, Wut, Aggressionen oder auch, dass die Jugendlichen von Gesprächspartnerinnen und -partnern von dem Thema Corona genervt seien. Was die Angst vor Ansteckungen betrifft, habe sich diese mit der Zeit etwas gelegt. Dagegen wurden andere Ängste, Unsicherheiten, Sorgen (Schule, Familie, Ausbildung etc.) und Perspektivlosigkeit sichtbar und zum Teil von niedergeschlagenen, trägen, bedrückten, deprimierten, befangenen Stimmungen gesprochen. Unter anderem wurde in einigen Interviews berichtet, dass der Drogenkonsum bei Jugendlichen gestiegen sowie Isolation, Vereinsamung und Depressionen ein Thema geworden seien.

Besonders von den Fachkräften hervorgehoben wurde das Bedürfnis und der Wunsch der Kinder und insbesondere der Jugendlichen nach Normalität. Dabei meint Normalität die Lebensweise vor Corona, besonders bezogen auf die Möglichkeit persönlicher Kontakte.

Insgesamt, so die Fachkräfte, freuten sich die meisten Kinder und Jugendlichen, als die Einrichtungen wieder öffneten.

\section{Der Digitalisierungsschub in der Offenen Kinder- und Jugendarbeit}

Eine Mehrheit der von uns untersuchten Einrichtungen war in der Lage, ab dem ersten Lockdown vom 23. März 2020 sehr schnell digitale Kontakte zu ihren Zielgruppen aufzubauen und zu erhalten. Und das war durchaus nicht selbstverständlich. Viele Einrichtungen haben zwar inzwischen Websites, aber direkte Kommunikation über soziale Medien und Messengerdienste war nicht die Regel. Stattdessen sahen und sehen viele Einrichtungen die leibhaftige Face-to-FaceBegegnung als zentrale Qualität der OKJA. Die Fähigkeit der Einrichtungen, sofort zu reagieren und auf digitale Kommunikation umzustellen, zeigt sich deutlich als eine der Stärken des Feldes in der Krise. Es wurden Livesendungen auf Instagram durchgeführt, ganze Jugendhäuser auf Discord elektronisch nachgestellt und belebt, Spiel- und Aktionsideen ausgetauscht und Sorgen und Fragen beantwortet. Einige Einrichtungen hatten jedoch auch Probleme mit dieser neuen Kommunikationsform: Es fehlte an Hardware und digitalen Kompetenzen. Immer wieder wurde auch (eher von den kommunalen Einrichtungen) über Verbote der Nutzung von sozialen Medien und Kommunikationsdiensten berichtet. Zudem gab 
und gibt es auch Zielgruppen (von benachteiligten oder jüngeren Kindern und Jugendlichen), denen es an den nötigen Endgeräten mangelt.

Aus unserer Sicht wurden die Fachkräfte durch Corona genötigt, endlich in großer Breite das Hauptmedium vieler Kinder und Jugendlichen in ihre Arbeit zu integrieren. Die bis dato häufig anzutreffende konzeptionelle Zurückhaltung und pädagogische Skepsis musste aufgegeben werden. Das führte auch zu neuen, positiven Erfahrungen: Man konnte überhaupt irgendwie mit Besucherinnen und Besuchern in Kontakt bleiben und die (oft von den Einrichtungen genannte) Botschaft rüberbringen: Ihr seid nicht allein und wir sind für euch da. Hinzu kam, dass die digitalen Medien eine Gegenseitigkeit erlauben; Kinder und Jugendliche können auch ihre Botschaften schicken, ihre kulturellen Handlungsstile präsentieren, Themen und Interessen artikulieren. Darauf können die Fachkräfte wieder mit ihrer Resonanz antworten und den Mediennutzerinnen und -nutzern Aufmerksamkeit und Anerkennung entgegenbringen. Man kann sich bestärken, informieren, präsentieren und austauschen. Das, was sonst an Kommunikation informell und en passant in der Einrichtung geschieht, tritt über die mediale Abbildung deutlicher hervor und kann reflexiver genutzt werden. Und manches, was im Alltag des offenen Bereichs mit seiner Gleichzeitigkeit und Fluidität schnell verloren geht, kann zumindest ein wenig und ein bisschen länger in der medialen Darstellung erkannt und berücksichtigt werden.

\section{Offene Kinder und Jugendarbeit Post-Corona}

Im Folgenden werden auf der Grundlage unseres Projekts einige Aspekte für die Weiterentwicklung der OKJA-Post-Corona-Zeit skizziert, die allerdings noch nicht auf der empirischen Grundlage der zurzeit laufenden Online-Befragung von Einrichtungen in NRW beruht. Besonders aus diesem Forschungsschritt werden wir weitere Schlussfolgerungen ziehen können.

\section{Strukturprinzipien zurückgewinnen}

Als Charakteristikum der OKJA werden immer wieder folgende Strukturprinzipien genannt: freiwillige Teilnahme, wechselnde Teilnahme, unterschiedliche Teilnehmer:innen, offene Ziele, Inhalte und Arbeitsweisen, geringe institutionelle Macht, Beziehungsabhängigkeit und Diskursivität (vgl. Sturzenhecker 2020). Diese Prinzipien erzeugen die strukturelle Grundlage der Offenheit der Institution und eröffnen Freiräume, in denen unterschiedlichste Besucher:innen mit ihren 
Fachkräften immer wieder neu Arbeitsbündnisse zu Inhalten und Handlungsweisen aushandeln und umsetzen können.

Nachdem im Lockdown vom März 2020 der Zugang zu den Einrichtungen nur noch durch digitale Kommunikation möglich war, wurden im Anschluss relativ restriktive Bedingungen für den Besuch der Einrichtungen eingerichtet, wie Anmeldungen, Eintragen von Namen und Adresse, Begrenzung der Besucher:innenzahl sowie Einschränkung der Bewegungsfreiheit in den Einrichtungen. Auch wenn sich die Bedingungen im Frühjahr und Sommer 2020 zum Teil etwas gelockert haben, sind die meisten Einrichtungen weit davon entfernt, ihre Rahmenbedingungen wieder so herzustellen, wie sie vor der Corona-Krise üblich waren, insbesondere was den offenen Bereich der Einrichtungen angeht. Die erhöhten Fallzahlen im Winter 2020 führten sogar wieder zu Schließungen von Einrichtungen.

- Die Strukturprinzipien müssen zurückgewonnen werden, gemeinsam mit Kindern und Jugendlichen.

- Die Freiraum-Funktion der Jugendarbeit muss stärker sichtbar gemacht werden als Alleinstellungsmerkmal der Offenen Kinder- und Jugendarbeit.

- Diese Freiraum-Erfahrung kennen viele Kinder und Jugendliche nicht mehr, sie muss wieder zurückgewonnen werden.

- Vielleicht muss das soziale Setting des Offenen Bereiches regelrecht wieder eingeübt werden?

\section{Sozialraumorientierung intensivieren}

Die untersuchten Einrichtungen hatten nicht zu allen Besucherinnen und Besuchern in ihrem Einzugsgebiet digitale Kontakte und nicht alle Kinder und Jugendlichen verfügten über die dafür nötige technische Ausstattung. Zudem sollte bei aller neuen Begeisterung für das Digitale doch der direkte persönliche Kontakt nicht aufgegeben werden. Das bedeutete, dass die Fachkräfte ihre Einrichtungen verlassen und hinaus in den Sozialraum gehen mussten. Das galt auch für Einrichtungen, die vorher nicht unbedingt mobil in ihren Stadtteilen oder aufsuchend unterwegs waren: Um den Kontakt zu Kindern und Jugendlichen zu halten, unternahmen die Fachkräfte Spaziergänge und Rundgänge, suchten Treffpunkte wie Spielplätze, Supermärkte, Unterstände, Bushäuschen und Ähnliches auf. Einrichtungen, die schon vor der Coronakrise mobil waren, konnten auf ihre Erfahrung zurückgreifen und diese Praxis weiter ausbauen.

- Nach der langen Phase in der Pandemie wird sich die Kinder- und Jugendkultur verändert haben. Die Jugendlichen kommen nicht einfach wieder, als wäre nichts geschehen. 


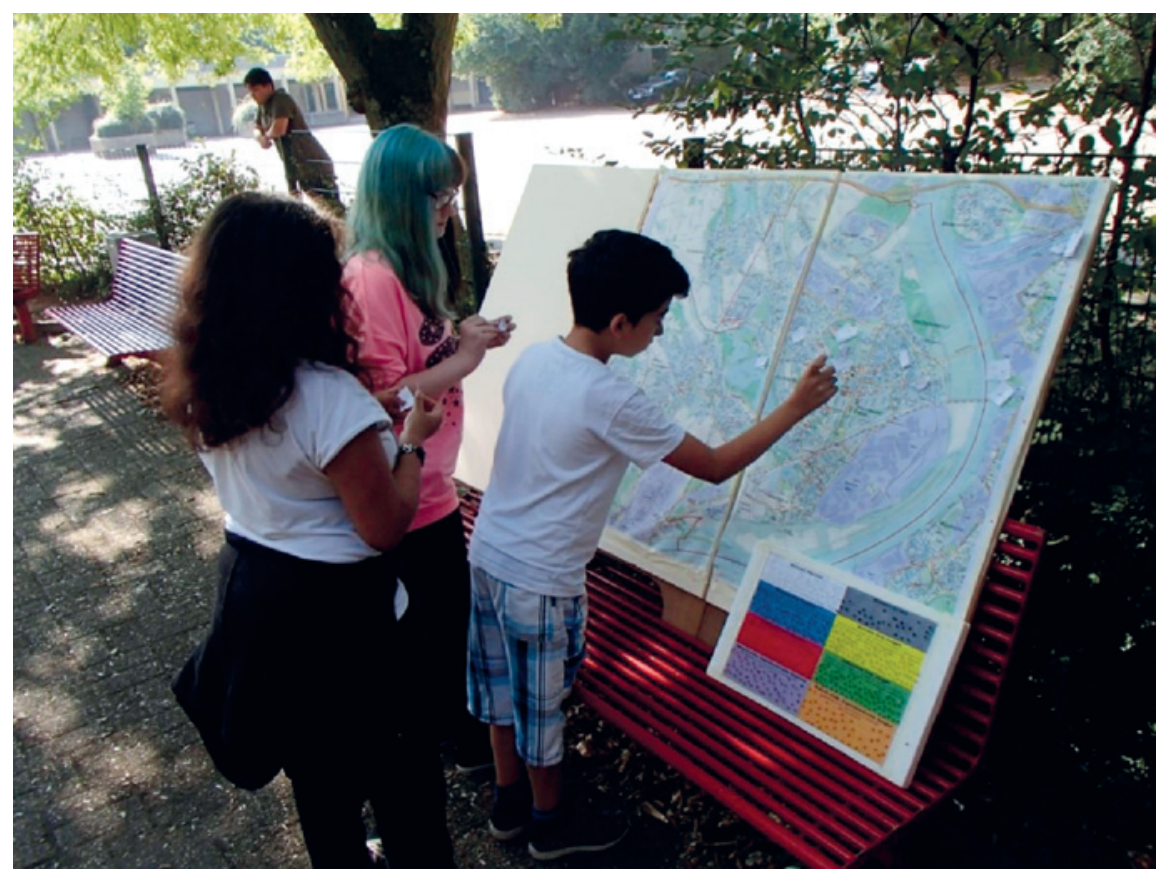

Abb. 1: Beispiel einer Sozialraumanalyse. Quelle: Ulrich Deinet

- Neue Sozialraum- und Lebensweltanalysen werden nötig sein, um (neue) Bedarfe zu ermitteln.

- Diese Analysen können mit Hilfe sozialräumlicher Methoden (z. B. Befragungen, Begehungen, Aktionen im öffentlichen Raum, herausreichende und mobile Arbeit) partizipativ, öffentlich sichtbar, aktivierbar gestaltet werden (siehe Abb. 1).

- Es geht um die Revitalisierung öffentlicher Räume mit Kindern und Jugendlichen...

- ...auch als kommunalpolitische Aufgabe der Beteiligung von Kindern und Jugendlichen an der Gestaltung des Zusammenlebens in der Kommune (Bezirks-Checks usw.)

\section{Kooperation von Jugendarbeit und Schule}

Insbesondere die Kooperationsprojekte mit Schulen sind nach dem Shutdown nur sehr zaghaft wiederaufgenommen worden. Ein Problem sind die völlig un- 
terschiedlichen Bedingungen im schulischen Bereich und in der OKJA, was die Gesundheits- und Abstandsregeln anbetrifft. Diese aus Sicht von Kindern und Jugendlichen, aber auch von Fachkräften völlig unverständliche Ungleichbehandlung zweier Bildungsbereiche führte in der Praxis zu absurden Situationen, wenn etwa eine Übermittag-Betreuung in einer Einrichtung als schulische Veranstaltung stattfinden konnte und die danach folgenden Angebote der Kinder- und Jugendarbeit nur in einem sehr eingeschränkten Maße.

Eine erst kurz vor der Corona-Krise beendete Studie (Deinet et al. 2020) stellte fest, dass sehr viele Angebote der Kooperation aktuell am Ort der Kinder- und Jugendarbeit stattfinden und nicht am Schulort. Dies hat mit den besonderen Bedingungen der Einrichtungen zu tun, die auch zum Teil von der Schule sehr geschätzt werden. Es stellt sich nun die Frage, wie diese Kooperation wiederhergestellt werden kann und wie die erreichte Öffnung von Schule auch in Richtung der Einrichtung der Offenen Kinder- und Jugendarbeit wieder realisiert werden kann.

- Wie geht es weiter in der Kooperation der OKJA mit der Institution Schule?

- Wie gestaltet OKJA Ihren Bezug zum Lebensort Schule, an dem sich Kinder und Jugendliche täglich viele Stunden aufhalten?

- Wie unterstützt die OKJA die „Schulbewältigung“ von Kindern und Jugendlichen nach der langen Zeit von Schulschließung? Viele Kinder und Jugendliche haben eine negative „School-Live-Balance“, die subjektiv ausgeglichen werden muss besonders auch in der Freizeit.

- Die Kooperation mit Schule muss neu verhandelt und gestaltet werden (auch in Bezug auf die neuen Förderungen durch den Bund: „Aktionsprogramm Aufholen nach Corona“).

- Kinder und Jugendliche brauchen in Bezug auf ihre schulische Situation beides: Unterstützung und wieder Freiraum.

\section{Selbstorganisation und Partizipation}

Offene Kinder- und Jugendarbeit kommt nur zustande, wenn die Kinder und Jugendlichen sich aktiv einbringen. Offene Jugendarbeit beruht auf einer alltäglichen Koproduktion, kommt im Alltag spontan zu gemeinsamen Entscheidungen, arbeitet in Projekten unter großer Mitbestimmung der Jugendlichen und findet auch zu demokratischen Strukturen der Partizipation an der Gestaltung der gemeinsamen Lebensführung in den Einrichtungen. Diese generelle Partizipation der Kinder und Jugendlichen war durch die Coronamaßnahmen verunmöglicht oder extrem erschwert. Die Schließungen zwangen die Fachkräfte, trotzdem aktiv zu werden, und zwar zunächst ohne Abstimmung mit den Kindern und Jugendlichen. Die Sicherheitsvorgaben des Landes, der Kommunen und der Träger führ- 
ten häufig dazu, dass die Fachkräfte deren Umsetzung komplett alleine bestimmten und in die Hand nahmen. Die durchaus begründeten und nachvollziehbaren staatlichen Eingriffe, die sich über die Fachkräfte bis zu den Kindern und Jugendlichen fortsetzten, produzieren aber auch das Risiko, die Bürger:innen, in unserem Fall die Kinder und Jugendlichen zu Objekten wohlmeinender Bestimmungen von oben zu machen.

Kinder und Jugendliche haben das schon im ersten Lockdown deutlich erfahren und auch kritisiert. Untersuchungen (z. B. Andresen et al. 2020) zeigen, dass viele junge Menschen beklagten, dass sie nur in ihrer Rolle als Zubeschulende gesehen wurden und zu ihren Meinungen und Vorschläge zum Umgang mit Corona (gerade auch in den pädagogischen Institutionen) zu wenig befragt und erst recht nicht gehört wurden. Viele gesellschaftliche Gruppen haben es geschafft, ihre Probleme angesichts der Coronaeinschränkungen öffentlichkeitswirksam zu präsentieren. Die nicht als gemeinsame Gruppierung erkennbaren und handlungsfähigen Kinder und Jugendlichen bekamen die schon aus der Vergangenheit bekannte negative Aufmerksamkeit der Erwachsenengesellschaft: Reflexhaft wurden die öffentlichen Präsentationen von Interessen als unverantwortliche Coronapartys gebrandmarkt (vgl. LAG 2020). Aber eine gerade auch kommunale, politische Auseinandersetzung über jugendliche Gesellungswünsche und eine demokratische Aushandlung von deren Umsetzung unter möglichst sicheren Bedingungen fanden so gut wie nicht statt.

- Mit der Schließung der Offenen Bereiche gingen viele niedrigschwellige Möglichkeiten der Partizipation verloren (z. B. Spieleverleih, Thekendienst usw.), die zurückgewonnen werden müssen.

- Partizipationsgremien sind lange ausgefallen und werden teilweise digitalisiert, in virtuellen Räumen wurden neue Formen der (E-)Partizipation ermöglicht, diese sind aber von der Medienkompetenz und technischen Ausstattung der Kinder und Jugendlichen abhängig. Der digitale Ausbau wird weitergehen und damit stellen sich auch Fragen, wie diese Kommunikationsform in die Partizipation einbezogen werden kann. Aber „live“ muss die Partizipation wiederhergestellt und ausgebaut werden, vielleicht für viele Kinder und Jugendliche eine neue Erfahrung.

- Demokratische Partizipation in den Einrichtungen muss übergehen in eine Stärkung der Selbstorganisation der Kinder und Jugendlichen.

Einerseits war die Corona Zeit eine große Herausforderung für die Offene Kinderund Jugendarbeit und ihre Besucher:innen. Die klassischen Qualitäten und Arbeitsweisen der OKJA waren nicht realisierbar. Besonders für die Kinder und Jugendlichen fehlte diese Erfahrung eines Freiraums für ungezwungene Gesellung und Selbstbildung zu eigenen Interessen und Themen. Andererseits hat die Offe- 
ne Kinder- und Jugendarbeit auch wichtige Entwicklungsanstöße enthalten, klassische und neue Arbeitsweisen zu verbinden und ihre Stärken und Potenziale so zu erweitern.

\section{Literatur}

Andresen S, Lips A, Möller R, Rusack T, Schröer W, Thomas S, Wilmes J (2020) Kinder, Eltern und ihre Erfahrungen während der Corona-Pandemie. Universitätsverlag Hildesheim, Hildesheim. https://hildok.bsz-bw.de/frontdoor/index/index/docld/1081. Zugriff am 27. Dezember 2020.

Deinet U, Icking M, Rehrs S (2020) Offene Kinder- und Jugendarbeit und Schule in NRW. socialnet Materialien. https://www.socialnet.de/materialien/29066.php. Zugriff am 28. Dezember 2020.

Deinet U, Sturzenhecker B (2021) Erster Zwischenbericht zum Forschungsprojekt: Neustart der Offenen Kinder- und Jugendarbeit in NRW in der Corona-Zeit. https://www.ew.unihamburg.de/einrichtungen/ew2/sozialpaedagogik/forschung/neustart-der-offenenkinder--und-jugendarbeit-in-nrw-waehrend-der-corona-krise.html. Zugriff am 8. Juni 2021.

LAG Mobile Jugendarbeit/Streetwork Baden-Württemberg e. V. (2020) Nach Stuttgart Mobile Jugendarbeit/Streetwork als Chance, Stuttgart. https://www.lag-mobil.de/ positionspapier-nach-stuttgart-mobile-jugendarbeit-streetwork-als-chance. Zugriff am 27. Dezember 2020.

Sturzenhecker B (2020) Demokratiebildung in der Kinder- und Jugendarbeit. In: Bollweg P, Buchna J, Coelen T, Otto HU (Hg) Handbuch Ganztagsbildung. Springer, Wiesbaden: 1263-1273.

\section{Weiterführende Literatur}

Deinet U (Hg) (2005) Sozialräumliche Jugendarbeit - Grundlagen, Methoden, Praxiskonzepte. VS Verlag für Sozialwissenschaften, Wiesbaden.

Lindner W (2014) Arrangieren, Kohlhammer, Stuttgart.

Schmidt H (2017) Arbeitsbeziehungen zwischen Hauptamtlichen und Jugendlichen in der Offenen Kinder- und Jugendarbeit. deutsche jugend, 5: 211-221.

Sturzenhecker B, Deinet U, von Schwanenflügel L, Schwerthelm M (2021) Offene Kinder- und Jugendarbeit angesichts der Corona-Krise. In: Deinet U, Sturzenhecker B, Schwanenflügel L, Schwerthelm M (Hg) Handbuch Offene Kinder- und Jugendarbeit. Springer, Wiesbaden: 2001-2029. 
